JOURNAL OF SYNCHROTRON RADIATION

ISSN 1600-5775

Received 12 January 2019

Accepted 2 May 2019

Edited by J. Grünert, European XFEL, Germany

${ }^{1}$ This article will form part of a virtual special issue containing papers presented at the PhotonDiag2018 workshop.

Keywords: X-ray free-electron lasers; polarization; dynamical theory of diffraction.

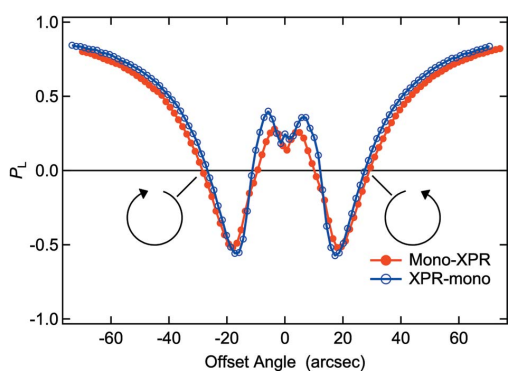

OPEN $\odot$ ACCESS

\section{Polarization control with an X-ray phase retarder for high-time-resolution pump-probe experiments at SACLA ${ }^{1}$}

\author{
Y. Kubota, ${ }^{a, b *}$ M. Suzuki, ${ }^{a}$ T. Katayama, ${ }^{a, b}$ K. Yamamoto, ${ }^{c}$ K. Tono, ${ }^{a, b}$ Y. Inubushi, ${ }^{a, b}$ \\ T. Seki, ${ }^{\mathrm{d}, \mathrm{e}}$ K. Takanashi, ${ }^{\mathrm{d}, \mathrm{e}}$ H. Wadati ${ }^{\mathrm{c}}$ and M. Yabashi ${ }^{\mathrm{b}, \mathrm{a} *}$
}

aJapan Synchrotron Radiation Research Institute (JASRI), 1-1-1 Kouto, Sayo-cho, Sayo-gun, Hyogo 679-5198, Japan, ${ }^{\mathbf{b}}$ RIKEN SPring-8 Center, 1-1-1 Kouto, Sayo-cho, Sayo-gun, Hyogo 679-5148, Japan, 'Institute for Solid State Physics, The University of Tokyo, 5-1-5 Kashiwanoha, Kashiwa, Chiba 277-8581, Japan, Institute for Materials Research, Tohoku University, 2-1-1 Katahira, Aoba-ku, Sendai, Miyagi 980-8577, Japan, and ${ }^{\mathbf{e}}$ Center for Spintronics Research Network, Tohoku University, 2-1-1 Katahira, Aoba-ku, Sendai, Miyagi 980-8577, Japan.

*Correspondence e-mail: kubota@spring8.or.jp, yabashi@spring8.or.jp

Control of the polarization of an X-ray free-electron laser (XFEL) has been performed using an X-ray phase retarder (XPR) in combination with an arrival timing diagnostic on BL3 of the SPring-8 Angstrom Compact free-electron LAser (SACLA). To combine with the timing diagnostic, a pink beam was incident on the XPR crystal and then monochromated in the vicinity of samples. A high degree of circular polarization of $\sim 97 \%$ was obtained experimentally at $11.567 \mathrm{keV}$, which agreed with calculations based on the dynamical theory of $\mathrm{X}$-ray diffraction. This system enables pump-probe experiments to be operated using circular polarization with a time resolution of 40 fs to investigate ultrafast magnetic phenomena.

\section{Introduction}

X-ray free-electron lasers (XFELs) based on the selfamplified spontaneous emission (SASE) principle (Kondratenko \& Saldin, 1980; Bonifacio et al., 1984) produce brilliant femtosecond light pulses with ultrahigh coherence in the X-ray region (Emma et al., 2010). The SPring-8 Angstrom Compact free-electron LAser (SACLA), constructed in Harima, Japan, is an XFEL facility with three beamlines: BL2 and BL3 for hard XFEL beams (Ishikawa et al., 2012; Yabashi et al., 2015), and BL1 for a soft XFEL beam (Owada, Togawa et al., 2018). Their pulse durations were evaluated to be $7.7 \mathrm{fs}$ on BL3 (Inubushi et al., 2012, 2017) and below 100 fs on BL1 (Kubota et al., 2019; Owada et al., 2019). Using arrival timing diagnostic systems between the XFEL and synchronized optical laser pulses, the time resolution in pump-probe experiments has been improved to less than 100 fs (Katayama et al., 2016; Owada, Nakajima et al., 2018; Owada et al., 2019).

Polarization is a fundamental property of light. In the $\mathrm{X}$-ray region, anisotropic and magnetic properties of matter have been widely investigated using various polarization states. A diamond X-ray phase retarder (XPR) (Hirano et al., 1991; Giles et al., 1994; Lang \& Srajer, 1995) has been installed on BL3 of SACLA to control the polarization of the hard XFEL beam (Suzuki et al., 2014) so as to study the ultrafast dynamics of chemical bonding and magnetic states. In particular, pumpprobe X-ray magnetic circular dichroism (XMCD) spectroscopy is a powerful method to investigate spin dynamics such as a demagnetization and magnetization reversal observed on 
a picosecond to femtosecond time scale (Kirilyuk et al., 2010; Takubo et al., 2017). Since the diamond XPR crystal has its diffraction plane rotated by $45^{\circ}$ from the horizontal, the horizontally polarized incident XFEL beam is decomposed into equal parts $\sigma$ - and $\pi$-polarized radiation relative to the diffraction plane. According to the dynamical theory of X-ray diffraction (Batterman \& Cole, 1964), the XPR crystal produces a phase retardation between these two components near the Bragg condition, and consequently one is able to control the polarization states. In a conventional configuration [which we call a 'mono-XPR' (or scheme A) configuration], a monochromatic beam with a bandwidth of $\Delta E / E \simeq 1 \times 10^{-4}$ produced with a perfect crystal monochromator is employed as the incident beam on the XPR crystal, because an energy bandwidth and/or angular divergence can cause degradation of the polarization states converted with the XPR crystal (Suzuki et al., 2014). However, one may consider locating the XPR crystal upstream of the monochromator [i.e. an 'XPRmono' (or scheme B) configuration] so as to expand the applicable range of experiments with various polarization states. For example, scheme B enables us to combine the XPR system with the arrival timing monitor (TM) on BL3 of SACLA, because the TM system requires the transport of a pink beam with a bandwidth of $\Delta E / E \simeq 5 \times 10^{-3}$ through the beamline optics (including the XPR crystal) located in the optics hutch $(\mathrm{OH})$ (Katayama et al., 2016).

In this study, we evaluated the degree of circular polarization $\left(P_{\mathrm{C}}\right)$ of the XFEL beam in scheme $\mathrm{B}$ that combines polarization control with the TM on BL3 of SACLA. We experimentally confirmed a high degree of $P_{\mathrm{C}}$, which is consistent with the calculated value and almost the same as that for scheme A (Suzuki et al., 2014).

\section{Experimental}

Fig. 1(a) shows the experimental setup with the XPR-mono (scheme B) configuration on BL3 of SACLA (Tono et al., 2013). In the OH, the pink XFEL beam reflected with mirrors was incident on the XPR crystal (Suzuki et al., 2014). In this study, a diamond (100) crystal $1.5 \mathrm{~mm}$ thick was used in a 220 symmetric Laue geometry. A branch beam of the XFEL, separated with a grating in the $\mathrm{OH}$, was used for the arrival TM in experimental hutch EH1 (Katayama et al., 2016). After passing through the XPR crystal, the main XFEL beam was monochromated at the Pt $L_{3}$ edge with a pair of channel-cut crystals $(\mathrm{CCs})$ in a $(+,-,-,+)$ geometry, which maintains the height of the beam, with $\mathrm{Si}(111)$ reflections installed in EH1.

For comparison, we set up the conventional mono-XPR (scheme A) configuration as shown in Fig. 1(b). A doublecrystal monochromator (DCM) with $\mathrm{Si}(111)$ reflections was used to produce the monochromatic beam. The monochromatic XFEL pulse was incident on the XPR crystal. The grating, TM and CCs monochromator were not used in this configuration.

In EH2, we installed two Kapton scattering beam monitors (BMs) to measure the intensities of the horizontal and vertical linear polarized components as shown in Fig. 1. An FePtPd (a) XPR-mono (scheme B) configuration

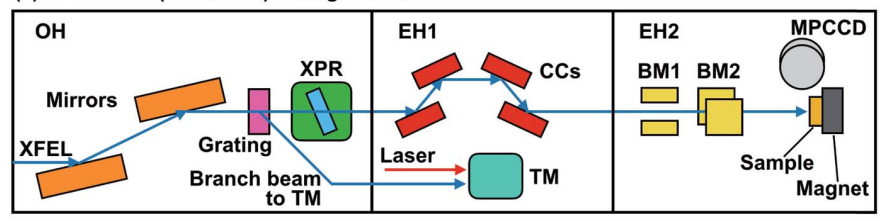

(b) Mono-XPR (scheme A) configuration

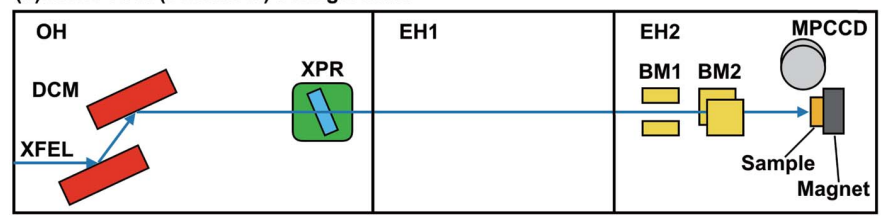

Figure 1

Schematic drawings of the experimental setup of $(a)$ the XPR-mono (scheme B) configuration and $(b)$ the mono-XPR (scheme A) configuration (see the main text) on BL3 of SACLA. XPR: X-ray phase retarder; TM: timing monitor; CCs: monochromator composed of channel-cut crystals; BM: beam monitor; MPCCD: multiport charge-coupled device; DCM: double-crystal monochromator.

film (50 nm thick) grown on an $\mathrm{MgO}(100)$ substrate by magnetron sputtering at $773 \mathrm{~K}$ was used to evaluate the $P_{\mathrm{C}}$ of the XFEL by measuring the XMCD. The sample forms an $L 1_{0}$ structure with an out-of-plane easy magnetization direction (Seki et al., 2011; Takubo et al., 2017). Using an Nd-Fe-B permanent magnet, an external magnetic field of $\mu_{0} H=$ $\pm 0.59 \mathrm{~T}$ was applied along the surface normal of the sample film to saturate the magnetization. The sample was maintained at room temperature during measurements. We used two pairs of the sample and the permanent magnet, with opposite field directions for each pair, to measure the XMCD signal. The two samples were cut from the same parent film, and the magnetic field strengths were prepared to be nearly the same for the two configurations. The remaining systematic errors in the magnetic asymmetry were corrected. The XFEL beam was incident on the sample in the surface normal direction. To measure the absorbed intensity at the Pt $L_{3}$ edge, we detected the Pt $L_{\alpha}(9.443 \mathrm{keV})$ fluorescence with a multiport chargecoupled device (MPCCD) detector (Kameshima et al., 2014), as shown in Fig. 1.

\section{Results and discussion}

Fig. 2(a) shows the intensities of the horizontal $\left(I_{x}\right)$ and vertical $\left(I_{y}\right)$ polarization components as a function of the offset angle of the XPR crystal from the 220 symmetric Laue geometry at $11.567 \mathrm{keV}$ detected with the Kapton scattering BMs in scheme B. This photon energy was selected so as to maximize the value of the XMCD. From the measured values of $I_{x}$ and $I_{y}$, the degree of linear polarization, $P_{\mathrm{L}}$, was determined using the equation

$$
P_{\mathrm{L}}=\frac{1}{Q} \frac{I_{x}-I_{y}}{I_{x}+I_{y}}
$$

where $Q$ is the correction factor of the BMs used in this experiment. The factor was estimated to be $Q=$ 

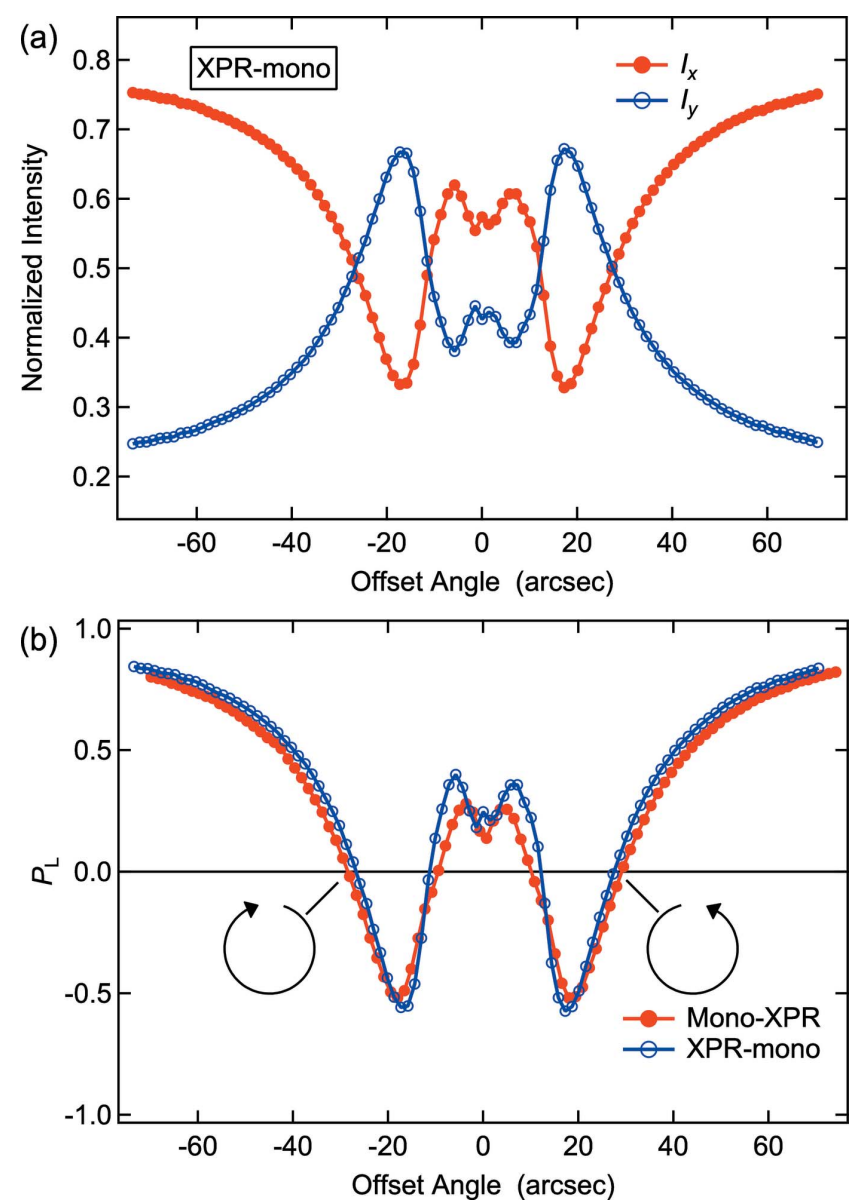

Figure 2

(a) The intensities of the horizontal $\left(I_{x}\right.$, red solid circles) and vertical $\left(I_{y}\right.$, blue open circles) polarization components as a function of the offset angle of the XPR crystal in the XPR-mono (scheme B) configuration at $11.567 \mathrm{keV}$. (b) The XPR crystal angle dependence of $P_{\mathrm{L}}$. The red solid circles and blue open circles represent the values of $P_{\mathrm{L}}$ in the mono-XPR (scheme A) and XPR-mono (scheme B) configurations, respectively.

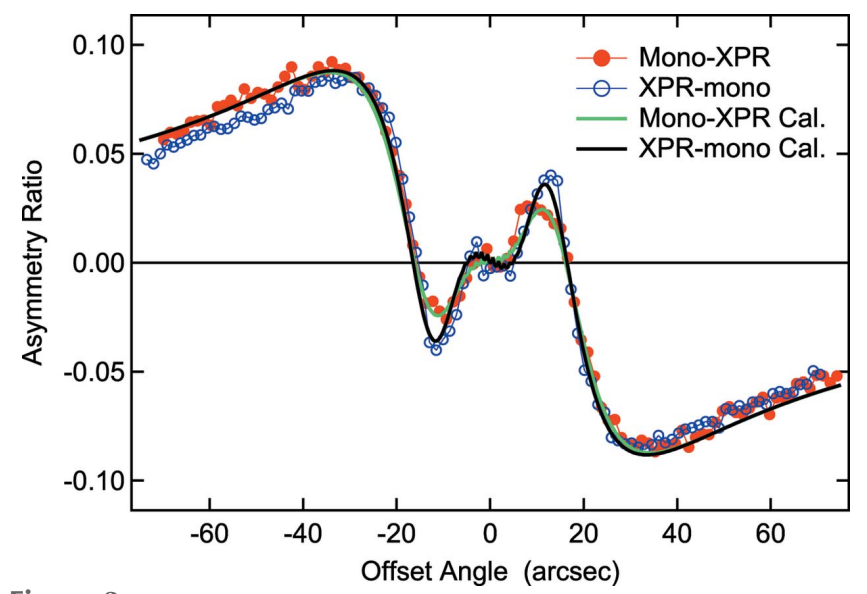

Figure 3

The magnetic asymmetry ratio obtained from the FePtPd film at the Pt $L_{3}$ edge as a function of the offset angle of the XPR crystal. The red solid circles and blue open circles represent the values in the mono-XPR (scheme A) and XPR-mono (scheme B) configurations, respectively. The green and black solid curves represent the calculated values for the scheme $\mathrm{A}$ and $\mathrm{B}$ configurations assuming Gaussian bandwidths of $\Delta E / E=1.1 \times 10^{-4}$ and $8.6 \times 10^{-5}$, respectively.
$0.5990 \pm 0.0005$ by a measurement of the intensity of the horizontally polarized component without the XPR crystal (Suzuki et al., 2014). Fig. 2(b) shows the XPR crystal angle dependence of $P_{\mathrm{L}}$. We also operated the same measurement in scheme A. One finds good agreement between the two configurations, which shows that polarization control with the XPR crystal works properly even for scheme B. At the points of $P_{\mathrm{L}}=0$, the XPR generates $\pm \pi / 2$ phase retardation, and we obtain circular polarization with right- and left-helicity, respectively. A small discrepancy between the two configurations is due to the difference between the bandwidths obtained with the CCs and DCM (Suzuki et al., 2014). The monochromatic beam generated with the CCs has a bandwidth about 0.8 times smaller than that obtained with the DCM due to the difference in their reflection geometries: $(+,-,-,+)$ for the CCs and $(+,-)$ for the DCM.

Next, we estimated $P_{\mathrm{C}}$ in scheme $\mathrm{B}$ by measuring the XMCD of the FePtPd film at the Pt $L_{3}$ edge. A magnetic asymmetry ratio, $R$, was determined by the equation

$$
R=\frac{I^{+} / I_{0}^{+}-I^{-} / I_{0}^{-}}{I^{+} / I_{0}^{+}+I^{-} / I_{0}^{-}}
$$

where $I^{+}\left(I^{-}\right)$is the absorbed intensity in the FePtPd film detected with the MPCCD for a magnetic field applied in the direction antiparallel (parallel) to the X-ray incident direction. $I_{0}^{+}\left(I_{0}^{-}\right)$is the intensity of the incident XFEL beam, which is the sum of the output of the BMs. Fig. 3 shows the values of $R$ as a function of the offset angle of the XPR crystal in schemes $\mathrm{A}$ and $\mathrm{B}$, with the calculation curves obtained by the same method as that used in a previous study (Suzuki et al., 2014). This result indicates that $P_{\mathrm{C}}$ in scheme $\mathrm{B}$ is almost the same as that of scheme A with a high value of 0.97 , which was obtained in the previous study (Suzuki et al., 2014). The uncertainty in $P_{\mathrm{C}}$ in scheme $\mathrm{B}$ was estimated to be \pm 0.06 . The discrepancy in the offset angles between -20 and $20 \mathrm{arcsec}$ is due to the influence of the bandwidth, which was seen in $P_{\mathrm{L}}$ (Suzuki et al., 2014). For negative offset angles, $R$ obtained in scheme $\mathrm{B}$ is lower than that in scheme A, which is probably due to a glitch as discussed in the previous study (Suzuki et al., 2014).

From the experiments, we confirmed that a high degree of $P_{\mathrm{C}}$ was retained in scheme $\mathrm{B}$ at $11.567 \mathrm{keV}$. However, according to the dynamical theory of X-ray diffraction (Batterman \& Cole, 1964), a CCs monochromator could introduce a phase difference $(\delta)$ between the $\sigma$ and $\pi$-polarization components, which leads to degradation of the polarization states produced with the XPR crystal. To investigate the effect of CCs on $P_{\mathrm{C}}$ over a wide photon energy range, we calculated the values of $P_{\mathrm{C}}$ based on the dynamical theory of diffraction. Since the angular divergence of the XFEL beam $(\sim 2 \mu \mathrm{rad})$ is sufficiently smaller than the Darwin width of the $\mathrm{Si}(111)$ reflection $(\sim 20 \mu \mathrm{rad})$, we only considered the effect of the energy spread. Fig. 4(a) shows rocking curves for the $\sigma$ - and $\pi$-polarization components and the resulting $\delta$ and $P_{\mathrm{C}}$ after the four-fold reflections of the CCs at $E_{0}=$ $11.567 \mathrm{keV}$. For the calculation of $P_{\mathrm{C}}$, we assumed that the incident XFEL beam has circular polarization with $P_{\mathrm{C}}=1$. Although $P_{\mathrm{C}}$ decreased at the edges of the rocking curves, the 

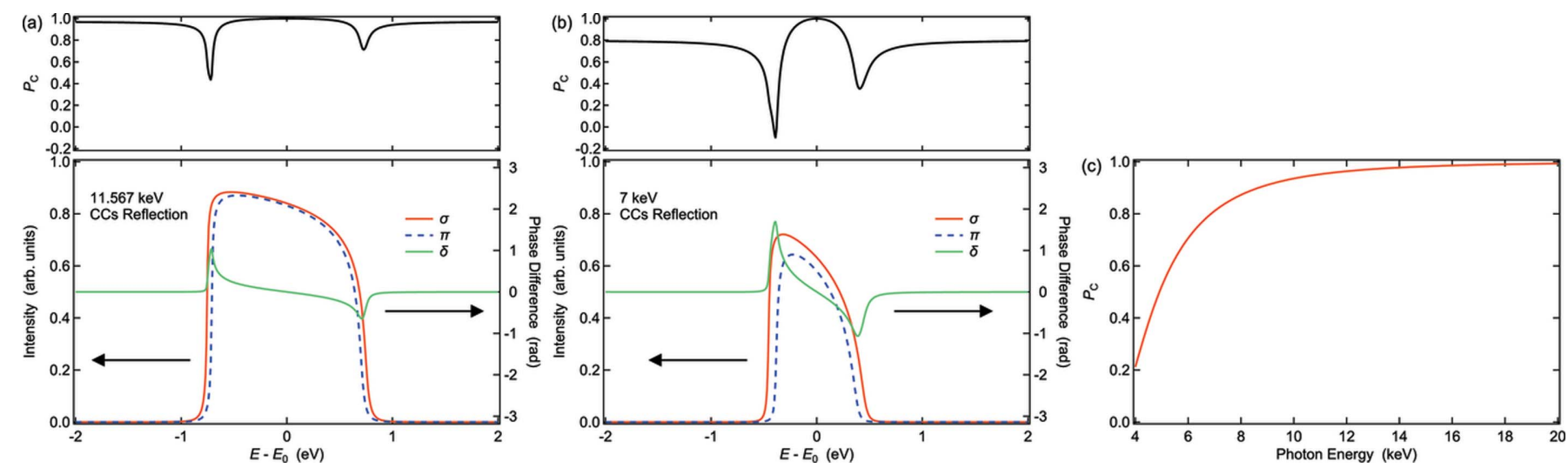

Figure 4

Calculated results based on the dynamical theory of X-ray diffraction. $(a, b)$ The values of $P_{\mathrm{C}}$ (top), and rocking curves with the additional phase difference $(\delta)$ between the $\sigma$ - and $\pi$-polarization components (bottom) after the four-fold reflections of the CCs monochromator as a function of relative photon energy $E-E_{0}$ at $(a) E_{0}=11.567 \mathrm{keV}$ and $(b) E_{0}=7 \mathrm{keV}$. The black solid lines represent the values of $P_{\mathrm{C}}$. The red solid and blue dashed lines represent rocking curves for the $\sigma$ - and $\pi$-polarization components (left axes), respectively. The green solid lines represent the values of $\delta$ (right axes). (c) Calculated values of $P_{\mathrm{C}}$ after the four-fold reflections of the CCs monochromator as a function of photon energy. The values of $P_{\mathrm{C}}$ were obtained by weighted averaging.

weighted average value was maintained at 0.96 at $11.567 \mathrm{keV}$. This result is consistent with our experimental results, showing that $P_{\mathrm{C}}$ in scheme $\mathrm{B}$ has almost the same value as that in scheme A. Note that the experimental value of $P_{\mathrm{C}} \simeq 0.97$ in scheme B included the effects of not only the degradation by the CCs but also the narrower bandwidth than that obtained by the DCM. Fig. 4(c) shows the weight-averaged values of $P_{\mathrm{C}}$ after the four-fold reflections of the CCs as a function of photon energy. This result shows that a high $P_{\mathrm{C}}$ can be maintained in a high photon energy region (larger than $\sim 10 \mathrm{keV}$ ), which includes XMCD for the $L$ edges of $5 d$ elements such as Pt, Ir and Os (Wienke et al., 1991; Schütz et al., 1989). On the other hand, the value of $P_{\mathrm{C}}$ decreases drastically in scheme $\mathrm{B}$ in a lower energy region of less than $\sim 10 \mathrm{keV}$, which includes the $K$ edges of $3 d$ transition metals and the $L$ edges of rare earth elements (Schütz et al., 1987, 1989; Giles et al., 1994). For example, Fig. 4(b) shows rocking curves with $\delta$ and the values of $P_{\mathrm{C}}$ after the four-fold reflections of the CCs at $E_{0}=7 \mathrm{keV}$. To improve $P_{\mathrm{C}}$ in this energy region, a single $\mathrm{CC}$ monochromator can be used in scheme $\mathrm{B}$. Alternatively, a high-intensity monochromatic beam generated with the self-seeded XFEL scheme (Amann et al., 2012; Lindberg \& Shvyd'ko, 2012; Inoue et al., 2019) is available for ultrafast magnetic measurements.

\section{Acknowledgements}

The authors thank Drs Kenji Tamasaku and Taito Osaka for valuable discussions. We would like to acknowledge the supporting members of the SACLA facility.

\section{Funding information}

This research was partially supported by the Japan Society for the Promotion of Science under a Grant-in-Aid for Scientific Research (B) (Grant No. 17H02823). The experiment was performed on BL3 of SACLA with the approval of the Japan Synchrotron Radiation Research Institute (JASRI) (proposal No. 2017B8088).

\section{References}

Amann, J., Berg, W., Blank, V., Decker, F. J., Ding, Y., Emma, P., Feng, Y., Frisch, J., Fritz, D., Hastings, J., Huang, Z., Krzywinski, J., Lindberg, R., Loos, H., Lutman, A., Nuhn, H. D., Ratner, D., Rzepiela, J., Shu, D., Shvyd'ko, Y., Spampinati, S., Stoupin, S., Terentyev, S., Trakhtenberg, E., Walz, D., Welch, J., Wu, J., Zholents, A. \& Zhu, D. (2012). Nat. Photon. 6, 693-698.

Batterman, B. W. \& Cole, H. (1964). Rev. Mod. Phys. 36, 681-717.

Bonifacio, R., Pellegrini, C. \& Narducci, L. M. (1984). Opt. Commun. 50, 373-378.

Emma, P., Akre, R., Arthur, J., Bionta, R., Bostedt, C., Bozek, J., Brachmann, A., Bucksbaum, P., Coffee, R., Decker, F., Ding, Y., Dowell, D., Edstrom, S., Fisher, A., Frisch, J., Gilevich, S., Hastings, J., Hays, G., Hering, P., Huang, Z., Iverson, R., Loos, H., Messerschmidt, M., Miahnahri, A., Moeller, S., Nuhn, H., Pile, G., Ratner, D., Rzepiela, J., Schultz, D., Smith, T., Stefan, P., Tompkins, H., Turner, J., Welch, J., White, W., Wu, J., Yocky, G. \& Galayda, J. (2010). Nat. Photon. 4, 641-647.

Giles, C., Malgrange, C., Goulon, J., de Bergevin, F., Vettier, C., Fontaine, A., Dartyge, E. \& Pizzini, S. (1994). Nucl. Instrum. Methods Phys. Res. A, 349, 622-625.

Hirano, K., Izumi, K., Ishikawa, T., Annaka, S. \& Kikuta, S. (1991). Jpn. J. Appl. Phys. 30, L407-L410.

Inoue, I., Osaka, T., Hara, T., Tanaka, T., Inagaki, T., Fukui, T., Goto, S., Inubushi, Y., Kimura, H., Kinjo, R., Ohashi, H., Togawa, K., Tono, K., Yamaga, M., Tanaka, H., Ishikawa, T. \& Yabashi, M. (2019). Nat. Photon. 13, 319-322.

Inubushi, Y., Inoue, I., Kim, J., Nishihara, A., Matsuyama, S., Yumoto, H., Koyama, T., Tono, K., Ohashi, H., Yamauchi, K. \& Yabashi, M. (2017). Appl. Sci. 7, 584.

Inubushi, Y., Tono, K., Togashi, T., Sato, T., Hatsui, T., Kameshima, T., Togawa, K., Hara, T., Tanaka, T., Tanaka, H., Ishikawa, T. \& Yabashi, M. (2012). Phys. Rev. Lett. 109, 144801.

Ishikawa, T., Aoyagi, H., Asaka, T., Asano, Y., Azumi, N., Bizen, T., Ego, H., Fukami, K., Fukui, T., Furukawa, Y., Goto, S., Hanaki, H., Hara, T., Hasegawa, T., Hatsui, T., Higashiya, A., Hirono, T., Hosoda, N., Ishii, M., Inagaki, T., Inubushi, Y., Itoga, T., Joti, Y., Kago, M., Kameshima, T., Kimura, H., Kirihara, Y., Kiyomichi, A., 
Kobayashi, T., Kondo, C., Kudo, T., Maesaka, H., Maréchal, X. M., Masuda, T., Matsubara, S., Matsumoto, T., Matsushita, T., Matsui, S., Nagasono, M., Nariyama, N., Ohashi, H., Ohata, T., Ohshima, T., Ono, S., Otake, Y., Saji, C., Sakurai, T., Sato, T., Sawada, K., Seike, T., Shirasawa, K., Sugimoto, T., Suzuki, S., Takahashi, S., Takebe, H., Takeshita, K., Tamasaku, K., Tanaka, H., Tanaka, R., Tanaka, T., Togashi, T., Togawa, K., Tokuhisa, A., Tomizawa, H., Tono, K., Wu, S., Yabashi, M., Yamaga, M., Yamashita, A., Yanagida, K., Zhang, C., Shintake, T., Kitamura, H. \& Kumagai, N. (2012). Nat. Photon. 6, 540-544.

Kameshima, T., Ono, S., Kudo, T., Ozaki, K., Kirihara, Y., Kobayashi, K., Inubushi, Y., Yabashi, M., Horigome, T., Holland, A., Holland, K., Burt, D., Murao, H. \& Hatsui, T. (2014). Rev. Sci. Instrum. 85, 033110.

Katayama, T., Owada, S., Togashi, T., Ogawa, K., Karvinen, P., Vartiainen, I., Eronen, A., David, C., Sato, T., Nakajima, K., Joti, Y., Yumoto, H., Ohashi, H. \& Yabashi, M. (2016). Struct. Dyn. 3, 034301.

Kirilyuk, A., Kimel, A. V. \& Rasing, T. (2010). Rev. Mod. Phys. 82, 2731-2784.

Kondratenko, A. M. \& Saldin, E. L. (1980). Particle Accel. 10, 207216.

Kubota, Y., Inoue, I., Togawa, K., Kinjo, R., Iwayama, H., Harries, J. R., Inubushi, Y., Owada, S., Tono, K., Tanaka, T., Hara, T. \& Yabashi, M. (2018). 16th International Conference on Megagauss Magnetic Field Generation and Related Topics (MEGAGAUSS 2018), 25-29 September 2018, Kashiwa, Japan (https://doi.org/ 10.1109/MEGAGAUSS.2018.8722659).

Lang, J. C. \& Srajer, G. (1995). Rev. Sci. Instrum. 66, 1540-1542.
Lindberg, R. R. \& Shvyd'ko, Y. V. (2012). Phys. Rev. ST Accel. Beams, 15, 050706.

Owada, S., Fushitani, M., Matsuda, A., Fujise, H., Sasaki, Y., Hikosaka, Y., Hishikawa, A. \& Yabashi, M. (2019). In preparation. Owada, S., Nakajima, K., Togashi, T., Kayatama, T. \& Yabashi, M. (2018). J. Synchrotron Rad. 25, 68-71.

Owada, S., Togawa, K., Inagaki, T., Hara, T., Tanaka, T., Joti, Y., Koyama, T., Nakajima, K., Ohashi, H., Senba, Y., Togashi, T., Tono, K., Yamaga, M., Yumoto, H., Yabashi, M., Tanaka, H. \& Ishikawa, T. (2018). J. Synchrotron Rad. 25, 282-288.

Schütz, G., Frahm, R., Wienke, R., Wilhelm, W., Wagner, W. \& Kienle, P. (1989). Rev. Sci. Instrum. 60, 1661-1665.

Schütz, G., Wagner, W., Wilhelm, W., Kienle, P., Zeller, R., Frahm, R. \& Materlik, G. (1987). Phys. Rev. Lett. 58, 737-740.

Seki, T., Iwama, H., Shima, T. \& Takanashi, K. (2011). J. Phys. D Appl. Phys. 44, 335001.

Suzuki, M., Inubushi, Y., Yabashi, M. \& Ishikawa, T. (2014). J. Synchrotron Rad. 21, 466-472.

Takubo, K., Yamamoto, K., Hirata, Y., Yokoyama, Y., Kubota, Y., Yamamoto, S., Yamamoto, S., Matsuda, I., Shin, S., Seki, T., Takanashi, K. \& Wadati, H. (2017). Appl. Phys. Lett. 110, 162401.

Tono, K., Togashi, T., Inubushi, Y., Sato, T., Katayama, T., Ogawa, K., Ohashi, H., Kimura, H., Takahashi, S., Takeshita, K., Tomizawa, H., Goto, S., Ishikawa, T. \& Yabashi, M. (2013). New J. Phys. 15, 083035 .

Wienke, R., Schütz, G. \& Ebert, H. (1991). J. Appl. Phys. 69, 61476149.

Yabashi, M., Tanaka, H. \& Ishikawa, T. (2015). J. Synchrotron Rad. 22, 477-484. 